OPEN ACCESS

Edited by:

Wenqing Xia,

Nanjing No. 1 Hospital, China

Reviewed by:

Xuan Wei,

Capital Medical University, China Vijaya Prakash Krishnan Muthaiah, University at Buffalo, United States Jyothilakshmi Vasavan,

Algonquin College, Canada

${ }^{*}$ Correspondence:

Bensheng Qiu

bqiu@ustc.edu.cn

Jun Yang

yangjunacup@126.com

Xiaochun Yu

yuxc@mail.cintcm.ac.cn

${ }^{\dagger}$ These authors have contributed equally to this work

Specialty section:

This article was submitted to Auditory Cognitive Neuroscience,

a section of the journal

Frontiers in Neuroscience

Received: 14 July 2021 Accepted: 03 December 2021

Published: 28 January 2022

Citation:

Wei Y, Zhang W, Li Y, Liu X, Zha B, Hu $S$, Wang $Y$, Wang $X$, $Y u X$, Yang $J$ and Qiu B (2022) Acupuncture Treatment Decreased Temporal Variability of Dynamic Functional Connectivity in Chronic Tinnitus.

Front. Neurosci. 15:737993. doi: 10.3389/fnins.2021.737993

\section{Acupuncture Treatment Decreased Temporal Variability of Dynamic Functional Connectivity in Chronic Tinnitus}

\author{
Yarui Wei ${ }^{1,2 t}$, Wanlin Zhang ${ }^{3 t}$, Yu Li ${ }^{1}$, Xiangwei Liu ${ }^{3}$, Bixiang Zha ${ }^{3}$, Sheng $\mathrm{Hu}^{1,4}$, \\ Yanming Wang ${ }^{1}$, Xiaoxiao Wang ${ }^{1}$, Xiaochun Yu ${ }^{5 *}$, Jun Yang ${ }^{3 *}$ and Bensheng Qiu${ }^{1 *}$ \\ ${ }^{1}$ Hefei National Lab for Physical Sciences at the Microscale and the Center for Biomedical Engineering, University of Science \\ and Technology of China, Hefei, China, ${ }^{2}$ Department of Magnetic Resonance Imaging, The First Affiliated Hospital \\ of Zhengzhou University, Zhengzhou, China, ${ }^{3}$ Department of Acupuncture and Rehabilitation, The First Affiliated Hospital \\ of Anhui University of Traditional Chinese Medicine, Hefei, China, ${ }^{4}$ School of Medical Information Engineering, Anhui \\ University of Traditional Chinese Medicine, Hefei, China, ${ }^{5}$ Institute of Acupuncture and Moxibustion, China Academy \\ of Chinese Medical Sciences, Beijing, China
}

Acupuncture is recommended for the relief of chronic tinnitus in traditional Chinese medicine, but the underlying neural mechanism remains unclear. The human brain is a dynamic system, and it's unclear about acupuncture's effects on the dynamic functional connectivity (DFC) of chronic tinnitus. Therefore, this study based on resting-state functional magnetic resonance imaging (fMRI) investigates abnormal DFC in chronic tinnitus patients and the neural activity change evoked by acupuncture treatment for tinnitus. In this study, 17 chronic tinnitus patients and 22 age- and sex-matched normal subjects were recruited, and their tinnitus-related scales and hearing levels were collected. The fMRI data were measured before and after acupuncture, and then sliding-window and k-means clustering methods were used to calculate DFC and perform clustering analysis, respectively. We found that, compared with the normal subjects, chronic tinnitus patients had higher temporal variability of DFC between the supplementary motor area and medial part of the superior frontal gyrus, and it positively correlated with hearing loss. Clustering analysis showed higher transition probability (TP) between connection states in chronic tinnitus patients, and it was positively correlated with tinnitus severity. Furthermore, the findings showed that acupuncture treatment might improve tinnitus. DFC between the posterior cingulate gyrus and angular gyrus in chronic tinnitus patients after acupuncture showed significantly decreased, and it positively correlated with the improvement of tinnitus. Clustering analysis showed that acupuncture treatment might promote chronic tinnitus patients under lower DFC state, and it also positively correlated with the improvement of tinnitus. This study suggests that acupuncture as an alternative therapy method might decrease the tinnitus severity by decreasing the time variability of DFC in chronic tinnitus patients.

Keywords: acupuncture, chronic tinnitus, functional magnetic resonance imaging, dynamic functional connectivity, clustering analysis 


\section{INTRODUCTION}

Tinnitus is defined as the perception of non-speech sound in the absence of an external acoustic stimulus. It is a common medical symptom that can be debilitating, and approximately 1 in 10 adults have had the experience of tinnitus (Bhatt et al., 2016; Yang et al., 2018). However, no effective drug therapy is available for the tinnitus (Baguley et al., 2013), and various treatment options are applied, including education and counseling, sound therapy, cognitive behavioral therapy (CBT), medications, dietary supplementation, and acupuncture (Tunkel et al., 2014; Lv et al., 2021).

Acupuncture is recommended for the relief of chronic tinnitus in traditional Chinese medicine. Although some studies reported that acupuncture had few effective treatments for tinnitus (Podoshin et al., 1991; Kim et al., 2012), recent studies showed an effective acupuncture treatment for tinnitus (Pang et al., 2019; Huang et al., 2021; Wang et al., 2021). The acupoints commonly used are Tinggong (SI 19), Tinghui (GB 2), Yifeng (TE 17), Shuaigu (GB 8), Ermigen (R 2), Fengchi (GB 20), Zhongzhu (TE 3), Luxi (TE 19), and Ermen (TE 21). Moreover, the researchers assessed the effect of deqi for patients who received acupuncture to alleviate tinnitus by the randomization procedure, large sample, and standardized protocol (Xie et al., 2014). Lin et al. (2019) explored factors influencing the efficiency of acupuncture in tinnitus patients and found that the combination of acupoints and the number of acupuncture sessions contributed to a considerably better outcome.

Some studies were aimed to explore the science evidence of acupuncture in the treatment of tinnitus patients. Due to the lack of objective marker of tinnitus, tinnitus-related scales were used to assess the effect of acupuncture (Jeon et al., 2012; Kim et al., 2017). The acupuncture's effects for tinnitus were associated with the improvement of cochlear blood flow via the infrared thermography test (Cai et al., 2019). A previous study also assessed the cochlear function in patients with tinnitus by analyzing otoacoustic emissions and found that the amplitude of otoacoustic emissions was increased after acupuncture (de Azevedo et al., 2007). Ethyl single-photon emission computed tomography ( ${ }^{99 m}$ Tc-ECD SPECT) was also used to investigate the effect of acupuncture on brain perfusion in tinnitus patients; however, no between-group brain perfusion differences were observed (Laureano et al., 2016). Therefore, in terms of neural correlate, the science evidence of acupuncture for treating tinnitus is lacking. The resolution of functional magnetic resonance imaging (fMRI) is better than that of SPECT, and $\mathrm{fMRI}$ is a non-invasive functional imaging method. The previous study explored the central mechanism of transauricular vagus nerve stimulation (taVNS) to normal human by fMRI and found a suitable taVNS site for potential tinnitus treatment (Peng et al., 2018).

It is noteworthy that both the appearance of tinnitus and the effect of acupuncture tightly associate with the central system. The researchers explored the functional anatomy of tinnitus and found plastic changes in multiple neural systems in tinnitus patients (Lockwood et al., 1998). The previous studies showed the abnormal brain activity among the auditory cortex and non-auditory regions (limbic system, subcortex regions, brainstem, and cerebellum) in tinnitus patients (Liu and Chen, 2012; Bauer et al., 2013; Chen et al., 2015a, 2017; Berlot et al., 2020; Marschall et al., 2021). Moreover, researchers found that acupuncture modulated the cerebral cortex, limbic system, brainstem, cerebellum, and subcortical gray structures of human brain and evoked the integrated response of these regions (Hui et al., 2000; Hui et al., 2005; Napadow et al., 2009). Manual acupuncture at acupoint $\mathrm{Hegu}$ (LI 4) produced prominent decreases of fMRI signals in the limbic system and subcortical structures and increases of fMRI signals in the somatosensory cortex (Hui et al., 2000).

Static functional connectivity (SFC) analysis based on resting state fMRI investigated abnormal functional connectivity (FC) among different brain areas in tinnitus patients (Lv et al., 2017; Feng et al., 2018; Lan et al., 2020). Researchers have found some intrinsic connectivity networks using the SFC analysis, such as default mode network (Greicius et al., 2009), ventral and dorsal attention networks (Fox et al., 2006), and salience network (Seeley et al., 2007). However, the SFC is based on the implicit assumption of spatial and temporal stationary of fMRI data, which is over simple for complex activities of the human brain (Allen et al., 2014). When the mental activity is unconstrained, dynamics are potentially even more prominent under the resting state. Evidences suggested that dynamic functional connectivity (DFC) supply us new information about abnormal FC on the brain of patients with various diseases (Demirtas et al., 2016; Guo et al., 2018; Li et al., 2019; Wang et al., 2020; Chen et al., 2020a,b). However, the abnormal DFC and the neural activity change after acupuncture treatment were unclear in tinnitus patients.

In the present study, we aimed to investigate acupuncture therapy's effects on the DFC of the tinnitus patients. We analyzed the temporal variability of DFC on chronic patients before and after acupuncture and classified and assessed the states of DFC using k-means clustering analysis (Allen et al., 2014; Wang et al., 2016; Li et al., 2019). To our best knowledge, this study is the first to explore abnormal DFC in chronic tinnitus patients and the neural mechanism of acupuncture treatment for tinnitus.

\section{MATERIALS AND METHODS}

\section{Participants}

To begin, we collected 33 tinnitus patients and 22 normal subjects from December 2017 to October 2019. In these patients, we excluded six acute tinnitus patients, and 10 tinnitus patients withdrew consent. Finally, 17 chronic tinnitus patients and 22 aged- and sexed-matched normal subjects were included in this study (Table 1). The inclusion criteria of chronic tinnitus patients were as follows: (i) between 18 and 70 years of age, right-handed; (ii) subjective neurological tinnitus, patients reported that they heard sound on the unilateral or bilateral tinnitus, no history of acupuncture treatment; (iii) no magnetic resonance contraindications; (iv) no medical history with congenital or hereditary disease, endocrine immune disease, mental or neurological system disease or the severe disease of heart, liver, or kidney, and so on. The exclusion criteria of 
TABLE 1 | The demographic data of chronic tinnitus patients and normal subjects.

\begin{tabular}{lcccc}
\hline & $\begin{array}{c}\text { Chronic tinnitus } \\
(\boldsymbol{n}=\mathbf{1 7})\end{array}$ & $\begin{array}{c}\text { Control group } \\
(\boldsymbol{n}=\mathbf{2 2})\end{array}$ & $\boldsymbol{\chi}^{\mathbf{2}}$ /t -value & $\boldsymbol{P}$-value \\
\hline Age (years) & $47.29 \pm 2.85$ & $46.82 \pm 2.37$ & -0.129 & 0.898 \\
Sex (M/F) & $7 / 10$ & $6 / 16$ & 0.834 & 0.283 \\
Handedness (R/L) & $17 / 0$ & $22 / 0$ & - & - \\
Tinnitus duration(years) & $8.38 \pm 2.99$ & - & - & - \\
Tinnitus location(L/R/B) & $4 / 3 / 10$ & - & - & - \\
\hline
\end{tabular}

$B$, bilateral; $F$, female; $L$, left; $M$, male; $R$, right.

chronic tinnitus patients were as follows: (i) left-handed; (ii) other subjective tinnitus, objective tinnitus (including mechanical or pulsatile tinnitus), or acute tinnitus; (iii) magnetic resonance contraindications; (iv) pregnant, breastfeeding, or menstruating; (v) medical history with congenital or hereditary disease, endocrine immune disease, mental or neurological system disease, or the severe disease of heart, liver, or kidney, and so on; (vi) the history of mental or neurological medicine. In addition, all patients were instructed to avoid starting other interventions. All subjects gave the informed consent, and this study was approved by the Ethics Committee of the First Affiliated Hospital of Anhui University of Traditional Chinese Medicine (ethics number: 2021AH-29).

\section{Tinnitus-Related Scales and Hearing Level}

The scales to assess tinnitus severity included tinnitus handicap inventory (THI) (Newman et al., 1996), tinnitus disturbance inventory (TDI) (Baguley et al., 2000), visual analog scales (VAS) (Lindberg et al., 1992), and Khalfa hyperacusis questionnaire (KHQ) (Khalfa et al., 2002). TDI also described the tinnitus loudness. TDI divides the degree of the tinnitus loudness into seven levels, namely zero to sixth levels. Zero level means no tinnitus, and the sixth level means that the tinnitus is extremely loud and unbearable. Pure tone audiometry was used to measure the hearing threshold for chronic tinnitus patients before and after acupuncture. Before acupuncture, tinnitus-related scales were collected for all chronic tinnitus patients with the data of hearing level for 15 of the patients; after acupuncture, tinnitusrelated scales were collected for 14 chronic tinnitus patients with the data of hearing level for 11 of the patients.

\section{Acupuncture Treatment}

Acupuncture treatment was performed by specialized and licensed acupuncturists (Jun Yang and Bixiang Zha). The disposable sterile needle of $0.35 \mathrm{~mm}$ in diameter and $40 \mathrm{~mm}$ in length (Suzhou Tianxie Acupuncture and Moxibustion Appliance Co., Ltd., China) was used for performing manual acupuncture. The 7 obligatory acupoints were SI 19, GB 2, TE 17, GB 8, R 2, GB 20, TE 3 on the ipsilateral side with tinnitus. Additional acupoints were selected according to meridian diagnosis and the patient's symptoms. After sterilization, the acupuncturist manipulated the needle within the tolerance of the subject until the subjects obtained the deqi sensation without sharp pain. After achieving deqi sensation, the needle was located on the acupoints for $30 \mathrm{~min}$. Chronic tinnitus patients received 12 sessions of acupuncture treatments over 4 weeks (3 times per week). Fourteen chronic tinnitus patients finished the 12 sessions of acupuncture treatment among 17 chronic tinnitus patients. The patients didn't receive other supplementary medicine, electroacupuncture, or moxibustion throughout the course of acupuncture.

\section{Data Acquirement}

All subjects were scanned using a 3.0 T MRI scanner (Discovery MR750, GE, United States) with an 8-channel receiver array head coil located in Information Science Laboratory Center of University of Science and Technology of China. Head motion and scanner noise were reduced using foam padding and earplugs. The subjects were instructed to lie on their back quietly with their eyes closed but not to fall asleep and avoid thinking of anything particular during the scanning. We collected the data of MRI for all chronic tinnitus patients before acupuncture and for 12 of the patients after acupuncture. Among the 12 patients, we collected the data of tinnitus-related scales for all chronic tinnitus patients and the data of hearing level for 11 of the patients before acupuncture, and the data of tinnitus-related scales for 11 of the patients and the data of hearing level for 10 of the patients after acupuncture. The data of MRI after acupuncture was conducted 2 days after a course of acupuncture treatment. Structure images were acquired with 3D T1 BRAVO sequence with the following settings: repetition time (TR)/echo time $(\mathrm{TE})=8,160 / 31.8 \mathrm{~ms}$, slice number $=188$, slice thickness $=1 \mathrm{~mm}$, slice gap $=0 \mathrm{~mm}$, flip angle $=12^{\circ}$, field of view $(F O V)=25.6 \times 25.6 \mathrm{~cm}^{2}$, number of averages $=1$, matrix size $=256 \times 256$, voxel size $=1 \times 1 \times 1$ $\mathrm{mm}^{3}$. Functional images were acquired transversely with gradient spin echo planar imaging sequence with the following settings: $\mathrm{TR} / \mathrm{TE}=2,400 / 30 \mathrm{~ms}$, slice number $=46$, slice thickness $=3 \mathrm{~mm}$, slice gap $=3 \mathrm{~mm}$, flip angle $=90^{\circ}$, FOV $=19.2 \times 19.2$ $\mathrm{cm}^{2}$, number of averages $=1$, matrix size $=64 \times 64$, voxel size $=3 \times 3 \times 3 \mathrm{~mm}^{3}$. A total of 217 volumes were collected, resulting in a total scan time of $520.8 \mathrm{~s}$.

\section{Data Preprocessing}

Data analyses were preprocessed using Data Processing Assistant for Resting-State fMRI (DPARSF) programs (Yan and Zang, 2010), which is based on Statistical Parametric Mapping (SPM8) ${ }^{1}$ and MATLAB (MathWorks). The first 5 volumes were discarded and the remaining 212 consecutive volumes were used for data analysis. Slice-timing and realignment for head motion correction were performed. Nuisance covariates were regressed [including Friston 24 head motion parameters (Friston et al., 1996) and white matter and cerebrospinal fluid signals]. Then, data were coregistered with the structural images, spatial normalized to the Montreal Neurological Institute template (resampling voxel size $=3 \times 3 \times 3 \mathrm{~mm}^{3}$ ), smoothed with an isotropic Gaussian kernel [full width at half maximum $(\mathrm{FWHM})=6 \mathrm{~mm}]$, and detrended and filtered $(0.01 \sim 0.08 \mathrm{~Hz})$. Image volumes with framewise displacement $(\mathrm{FD})>0.2 \mathrm{~mm}$

\footnotetext{
${ }^{1}$ http://www.fil.ion.ucl.ac.uk/spm
} 
were scrubbed to reduce the effect of head motion using spline interpolation (He et al., 2018).

\section{Regions of Interest Selection and Static Functional Connectivity Network Analysis}

We used the automated anatomical labeling (AAL) template to calculate FC based on region of interest (ROI) analysis. A total of 90 ROIs of AAL template (without cerebellum) were divided into six main regions (including prefrontal regions, other regions of frontal lobe, parietal regions, occipital regions, temporal regions, and subcortical regions) (Tzourio-Mazoyer et al., 2002).

The average time courses were extracted from 90 ROIs for calculating SFC. Later, we calculated Pearson's correlation coefficients between each pair of the averaged time course in 90 ROIs, then Fisher $z$-transformation was used to convert $r$ values into $z$ values to improve the normality of correlation distribution.

\section{Dynamic Functional Connectivity Network Analysis}

Sliding window approach was widely used to measure brain DFC network for each subject (Allen et al., 2014; Zalesky et al., 2014). Here, we constructed a sliding temporal window (Hamming window) of 12 TRs. Following that, this window was used to slide on the time course with a step of 1 TR (2.4 s). In total, 201 (212$12+1$ ) temporal windows were produced. We then calculated FC in accordance with SFC analysis in each window, resulting in a time series of FC matrices $(90 \times 90$, Fisher $z$-transformed $)$ for the next analysis.

Unlike SFC, DFC reflected time-varying FC network. Therefore, we employed three common metrics to describe its dynamic characteristics: DFC-Str (strength) (Liu et al., 2018), DFC-SD (standard deviation) (Li et al., 2019), and ALFF-FC (amplitude of low frequency fluctuation of FC) (Han et al., 2011). Different metrics might reflect different temporal variability of DFC and complement each other. DFC-Str is the average of DFC, and DFC-SD is the standard deviation for the time series of FC matrices. ALFF-FC is a measure assessing the fluctuation of FC within the low-frequency range. A high-pass filtering for the original BOLD signals and a low-pass filtering for correlation coefficient time series with the cut-off frequency $1 / w$ are suggested to remove spurious fluctuations in DFC, when a certain window size $w$ is given (Leonardi and Van De Ville, 2015). Hence, for a given window size $w(12 \times 2.4 \mathrm{~s}=28.8 \mathrm{~s})$, we highpass filtered the ROI signals with cut-off frequency $1 / w$ prior to calculation of connectivity matrices, and then low-pass filtered the correlation coefficient time series with cut-off frequency $1 / w$ (Qin et al., 2015). Accordingly, ALFF-FC values were calculated within the frequency band of DFC from 0 to $1 / w$.

\section{Clustering Analysis}

To estimate the frequency and structure of DFC patterns, we applied a k-means algorithm (Lloyd, 1982) with the L1 distance function (Manhattan distance) (Allen et al., 2014) to cluster DFC data. For each subject, 201 windows $\times 4,005$ features were offered to the clustering analysis. We performed twice clustering analysis, one for chronic tinnitus patients before acupuncture and normal subjects and one for chronic tinnitus patients before and after acupuncture. Similar to EEG microstate analysis (Pascual-Marqui et al., 1995), for reducing redundancy between time windows (the chosen time step of 1 TR induces high autocorrelation in FC time series) and decreasing computational load, we performed subsampling across the temporal dimension to identify windowed covariance matrices with local maxima in FC variance (Allen et al., 2014; Wang et al., 2016). This yielded 1,435 exemplars [mean: $1,435 /(17+22)=36.8$ windows per subjects; range: $29 \sim 44]$ for the first clustering analysis and 867 exemplars [mean: $867 /(12+12)=36.1$ windows per subjects; range: $29 \sim 42]$ for the second clustering analysis, then $\mathrm{k}$-means were adopted for these exemplars with 500 repetitions to escape local minima, and the centroids produced from clustering exemplars were used to initialize clustering of all data (chronic tinnitus patients before acupuncture and normal subjects: $[(17+22)$ subjects $\times 201$ windows $=7,839$ instances; chronic tinnitus patients before and after acupuncture: $(12+12)$ subjects $\times 201$ windows $=4,824$ instances]. Finally, the optimal number of clusters ranging from 2 to 20 was estimated according to the elbow criterion (Liu et al., 2017), and the cluster medians (reshaped to matrix) were regarded as the FC state.

\section{State Analysis}

To compare state configuration between groups and within group, we computed one metric between states: transition probability (TP) (Li et al., 2019). TP was computed as the probability of transitions from one state to other state or lasting in the same state. When $\mathrm{N}$ cluster centers are calculated in the previous step, there will be $\mathrm{N} \times \mathrm{N}$ kinds of transition between states.

\section{Statistical Analysis}

To identify whether the demographic and scale data of each group were normally distributed, we performed Shapiro-Wilk's test. Results showed TDI scores were not normally distributed in chronic tinnitus before acupuncture, other demographic and scale data of each group were normally distributed. Two-sample $t$-tests and $\chi^{2}$-tests were used to compare demographic data between chronic tinnitus patients and normal subjects, and paired Student's $t$-tests was used to compare the effective of acupuncture treatment within chronic tinnitus patients before and after acupuncture with SPSS software. Wilcoxon-SignedRank test was used to compare TDI scores within chronic tinnitus patients before and after acupuncture. For SFC values and all metrics extracted from DFC (including DFC-Str, DFCSD, ALFF-FC, and TP), we performed a general linear model (GLM) to evaluate between-group and within-group differences in age, sex, and mean FD as covariates in the GRETNA toolbox (Wang et al., 2015). Afterward, multiple comparison correction (false discovery rate, FDR) was performed for connection metrics (SFC, DFC-Str, DFC-SD, and ALFF-FC). We calculated Pearson's correlation coefficients to perform correlation analysis between tinnitus-related scales scores (excluding TDI that used Spearmen's correlation) or hearing level and significant results between groups or within group. 


\section{RESULTS}

\section{Tinnitus Severity and Hearing Level Before and After Acupuncture}

The scores of TDI and VAS were significantly decreased in chronic tinnitus patients after acupuncture compared with before acupuncture (TDI: $Z=-2.251, P<0.05$; VAS: $T=-3.040$, $P<0.01$, Table 2). Any other significant difference was found in neither other tinnitus-related scales nor hearing level in chronic tinnitus patients after acupuncture compared with before acupuncture (Table 2).

\section{Static Functional Connectivity}

No significant difference for SFC was found in chronic tinnitus patients before acupuncture compared with normal subjects after FDR correction. SFC between the left posterior cingulate cortex (PCC) and right angular gyrus (ANG) in chronic tinnitus

TABLE 2 | Tinnitus severity and hearing level before and after acupuncture.

\begin{tabular}{lcccc}
\hline & Pre-acupuncture & Post-acupuncture & T/Z-value & $P$-value \\
\hline $\begin{array}{l}\text { Left ear } \\
\text { (dB/SPL, } n=11)\end{array}$ & $30.45+3.96$ & $29.89+3.30$ & -0.429 & 0.677 \\
Right ear & $38.21+7.63$ & $39.25+6.78$ & 0.599 & 0.562 \\
$(\mathrm{~dB} / \mathrm{SPL}, n=11)$ & & & & \\
$\mathrm{THI}(n=14)$ & $52.00+7.14$ & $46.71+7.25$ & -1.352 & 0.199 \\
$\mathrm{TDI}(n=14)$ & $3.07+0.51$ & $2.43+0.36$ & -2.251 & $0.024^{*}$ \\
$\operatorname{VAS}(n=14)$ & $5.64+0.59$ & $4.50+0.50$ & -3.040 & $0.009^{*}$ \\
$\mathrm{KHQ}(n=14)$ & $48.43+6.17$ & $43.36+5.95$ & -1.533 & 0.149
\end{tabular}

$\mathrm{dB}$, deci Bel; KHQ, Khalfa hyperacusis questionnaire; SPL, sound pressure level; TDI, tinnitus disturbance inventory; THI, tinnitus handicap inventory; VAS, visual analog scales. ${ }^{*} P<0.05$. patients after acupuncture was decreased compared with before acupuncture ( $T=-8.03, P=0.0000114$, Figure 1). No significant correlation was found between SFC of the left PCC and right ANG and tinnitus-related scales scores or hearing level.

\section{Dynamic Functional Connectivity Chronic Tinnitus Patients Before Acupuncture and Normal Subjects}

Based on two metrics (DFC-SD and ALFF-FC) to describe the time variability of DFC, higher DFC between the left supplementary motor area (SMA) and medial part of the left superior frontal gyrus (SFGmed) was found in chronic tinnitus patients before acupuncture than that in normal subjects (Table 3 and Figure 2). Based on the metrics (DFC-Str), no significant different DFC was found in chronic tinnitus patients before acupuncture than that in normal subjects.

To further explore the relationship between tinnitus severity and temporal variability of DFC in chronic tinnitus patients, we correlated tinnitus-related scales scores and hearing level with significant results from DFC-SD and ALFF-FC using the Pearson's or Spearman's (TDI scores) correlation coefficient. ALFF-FC between the left SMA and left SFGmed positively correlated with the hearing level of the right ear in chronic tinnitus patients before acupuncture $(r=0.571, P=0.026$, uncorrected, Figure 3). No other significant correlation was found between DFC of the left SMA and left SFGmed and other tinnitus-related scales or hearing level.

\section{Chronic Tinnitus Patients Before and After Acupuncture}

Based on the metric (DFC-Str) to describe DFC, DFC between the left PCC and right ANG was decreased in chronic tinnitus patients after acupuncture compared with before acupuncture

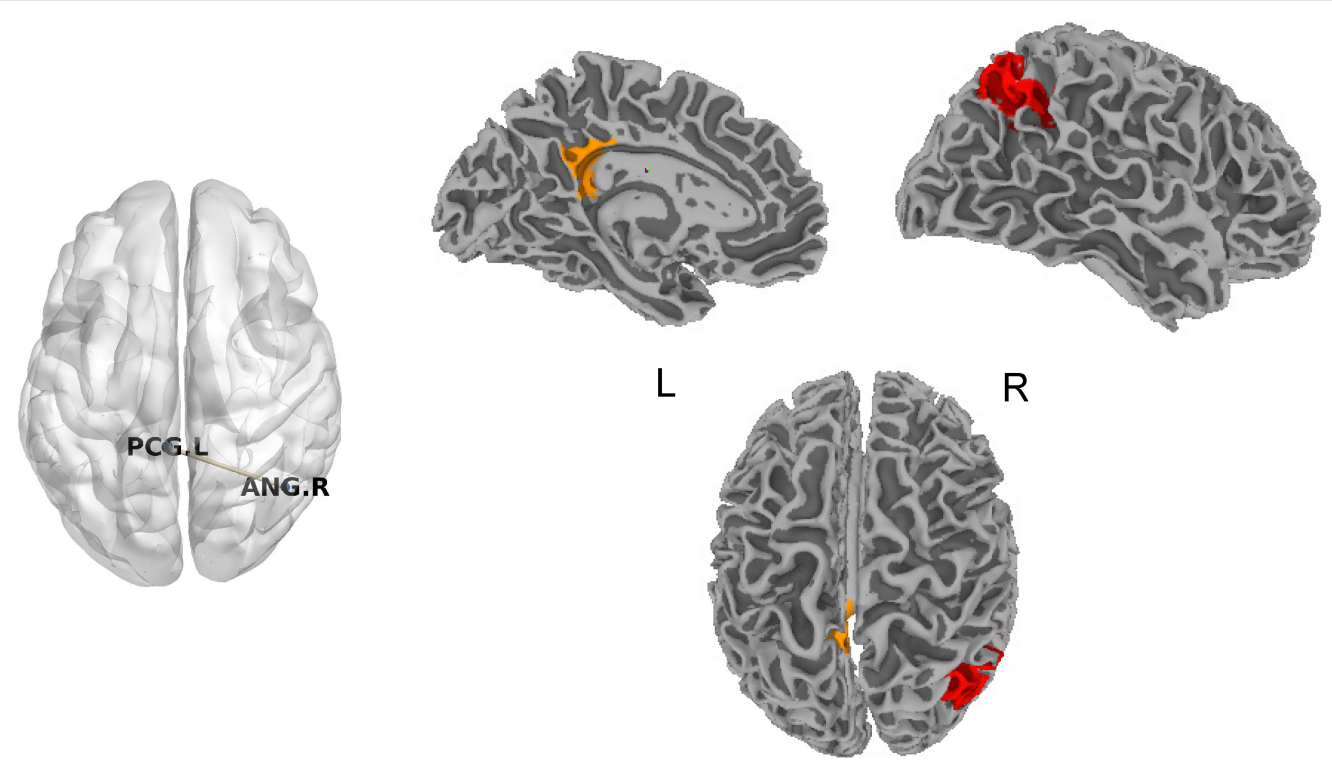

FIGURE 1 | Significant difference for SFC in chronic tinnitus patients before and after acupuncture. ANG, angular gyrus; L, left; PCG, posterior cingulate gyrus; R, right; SFC, static functional connectivity. 
TABLE 3 | Comparisons of temporal variability between chronic tinnitus patients before acupuncture and normal subjects.

\begin{tabular}{llll}
\hline DFC parameter & DFC pair & $\boldsymbol{T}$-value & $\boldsymbol{P}$-value \\
\hline DFC-SD & SMA.L-SFCmed.L & 5.42 & 0.0000074 \\
ALFF-FC & SMA.L-SFCmed.L & 5.24 & 0.0000106 \\
\hline
\end{tabular}

ALFF, amplitude of low frequency fluctuation; DFC, dynamic functional connectivity; FC, functional connectivity; L, left; SD, standard deviation; SFGmed, superior frontal gyrus, medial part; SMA, supplementary motor area.
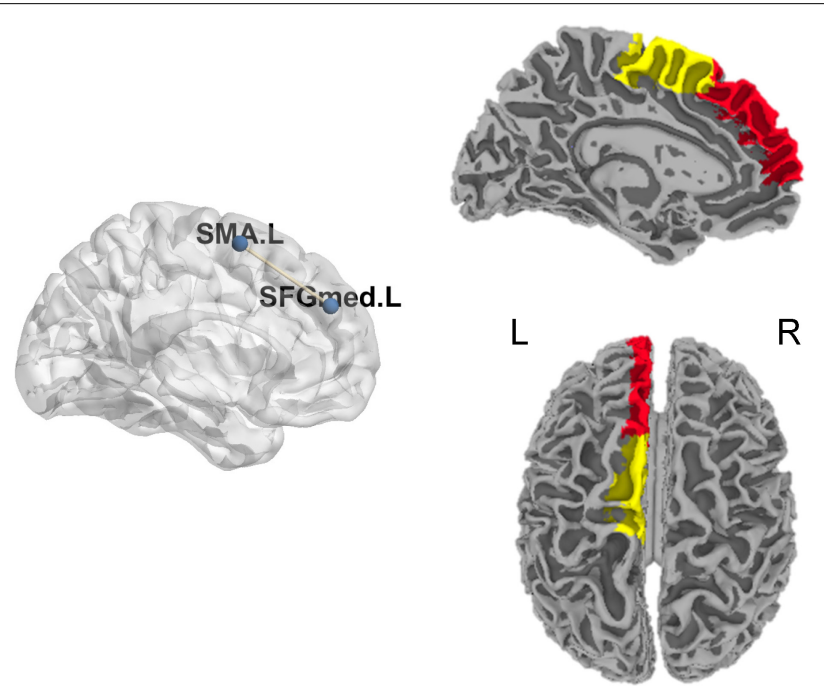

FIGURE 2 | Significant difference for DFC between chronic tinnitus patients before acupuncture and normal subjects. DFC, dynamic functional connectivity; L, left; R, right; SFGmed, superior frontal gyrus, medial part; SMA, supplementary motor area.

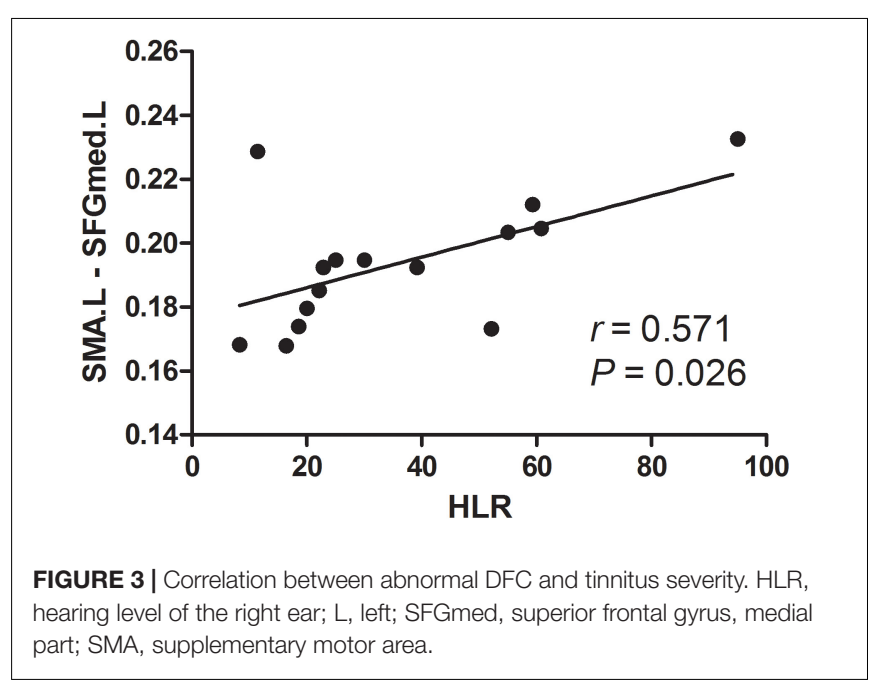

( $T=-8.96, P=0.0000043$, Figure 4). Based on the metrics (DFC-SD and ALFF-FC) to describe the time variability of DFC, no significant difference for DFC was found in chronic tinnitus patients after acupuncture compared with before acupuncture.

To further explore the relationship between tinnitus severity and temporal variability of DFC in chronic tinnitus patients before and after acupuncture, we correlated tinnitus-related scales scores and hearing level with significant results from DFCStr using the Pearson's or Spearman's (TDI scores) correlation coefficient. The decreased DFC-Str between the left PCC and left ANG positively correlated with the decreased TDI scores in chronic tinnitus patients before and after acupuncture $\left(r_{s}=0.623\right.$, $P=0.044$, uncorrected). No other significant correlation was found between DFC-Str of the left PCC and left ANG and other tinnitus-related scales scores or hearing level.

\section{Clustering and State Analysis Chronic Tinnitus Patients Before Acupuncture and Normal Subjects}

Based on elbow criterion, four clusters $(\mathrm{k})$ were determined in a search window ranging from 2 to 20 (Figures 5A,B). For each pair condition, we compared TP between groups. Compared with normal subjects, we found that the TP from state 2 to state 3 in chronic tinnitus patients before acupuncture was higher ( $T=2.303, P=0.028$, uncorrected, Figure 5C).

Meanwhile, for exploring the association between state configuration and tinnitus severity in chronic tinnitus patients before acupuncture, we performed Pearson or Spearman's (TDI scores) correlation analysis between TP from state 2 to state 3 and tinnitus-related scales scores or hearing level. As shown in Figure 6, TP from state 2 to state 3 in chronic tinnitus patients before acupuncture positively correlated with tinnitus severity (THI: $r=0.490, P=0.046$, uncorrected; VAS: $r=0.502, P=0.040$, uncorrected; KHQ: $r=0.564, P=0.018$, uncorrected). No other significant correlation was found between TP from state 2 to state 3 and other tinnitus-related scales scores or hearing level.

\section{Chronic Tinnitus Patients Before and After Acupuncture}

Based on elbow criterion, four clusters (k) were determined in a search window ranging from 2 to 20 (Figures 7A,B). For each pair condition, we compared TP within chronic tinnitus patients before and after acupuncture. Compared with before acupuncture, we found that the TP from state 3 (hyper-connected pattern) to state 1 (hypo-connected pattern) in chronic tinnitus patients after acupuncture was increased $(T=2.586, P=0.027$, uncorrected, Figure 7C).

For exploring the association between state configuration and tinnitus severity in chronic tinnitus patients before and after acupuncture, we performed Pearson's or Spearman's (TDI scores) correlation analysis between TP from state 3 to state 1 and tinnitus-related scales scores. Combining the data in chronic tinnitus patients before and after acupuncture, TP from state 3 to state 1 negatively correlated with TDI scores $\left(r_{s}=-0.416\right.$, $P=0.048$, uncorrected). No other significant correlation was found between TP from state 3 to state 1 and other tinnitusrelated scales scores or hearing level.

\section{DISCUSSION}

This study explores the brain functional network of chronic tinnitus patients and the neural mechanism of acupuncture 

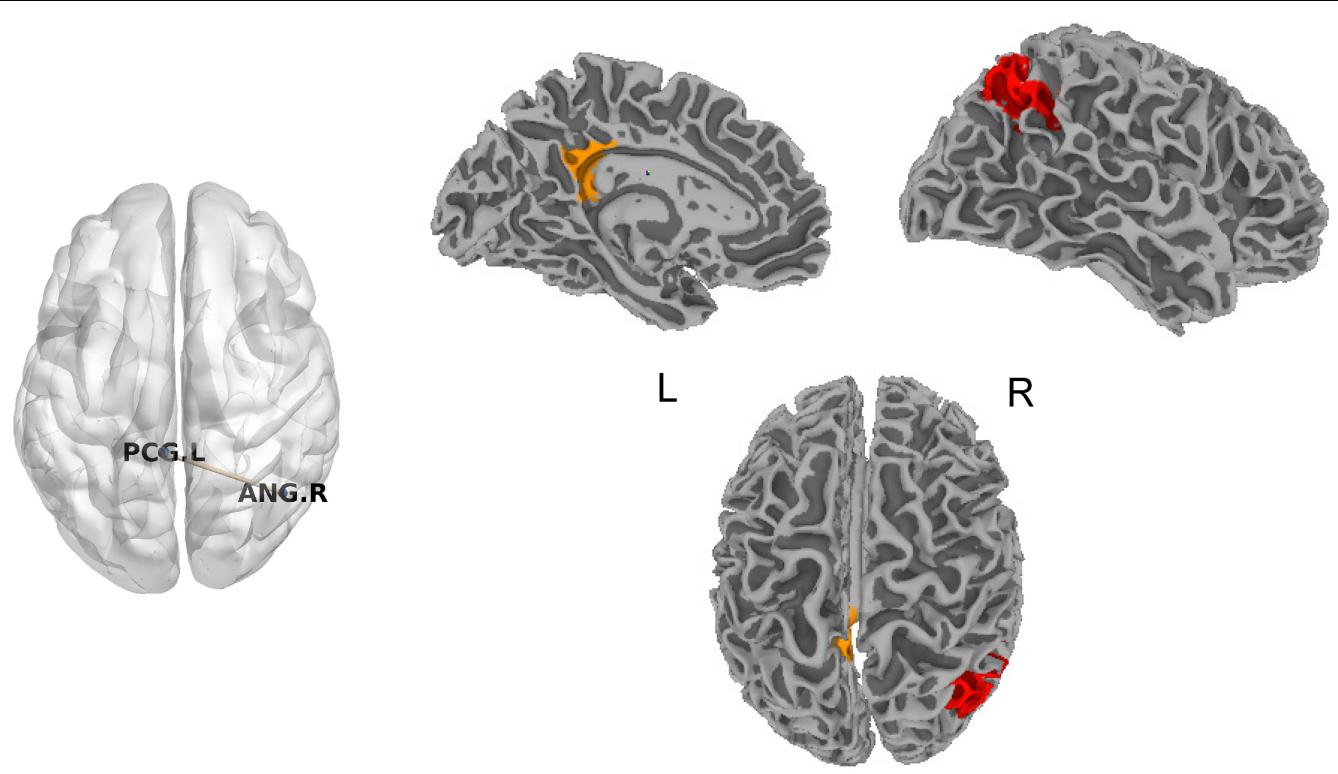

FIGURE 4 | Significant difference for DFC in chronic tinnitus patients before and after acupuncture. ANG, angular gyrus; DFC, dynamic functional connectivity; L, left; $P C G$, posterior cingulate gyrus; $R$, right.

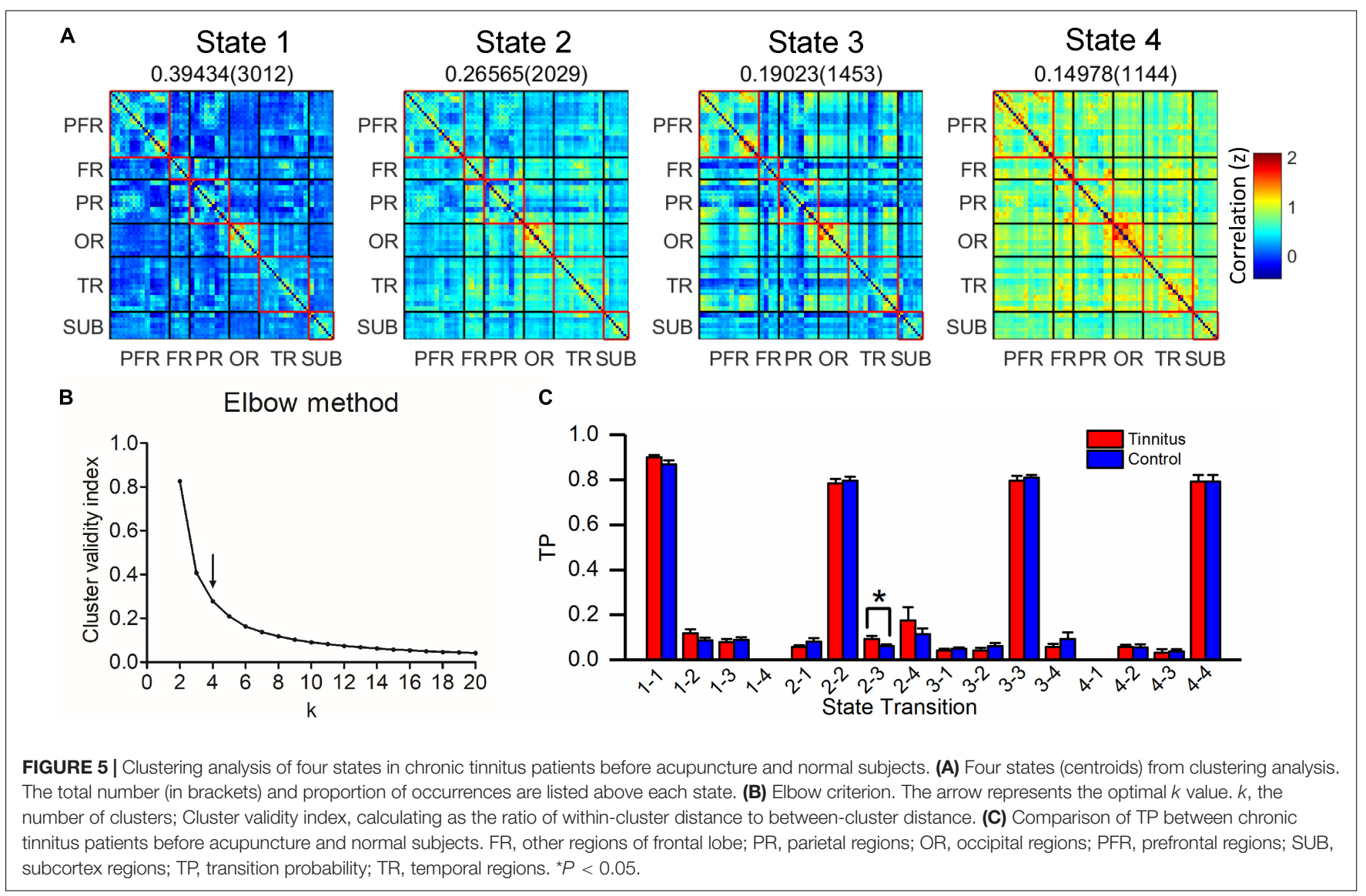

treatment for chronic tinnitus. The findings suggest that acupuncture might improve tinnitus by decreasing the temporal variability of DFC of the patients. The result showed that, compared with normal subjects, chronic tinnitus patients suffered a higher temporal variability of DFC between the left SMA and left SFGmed, which positively correlated with hearing 

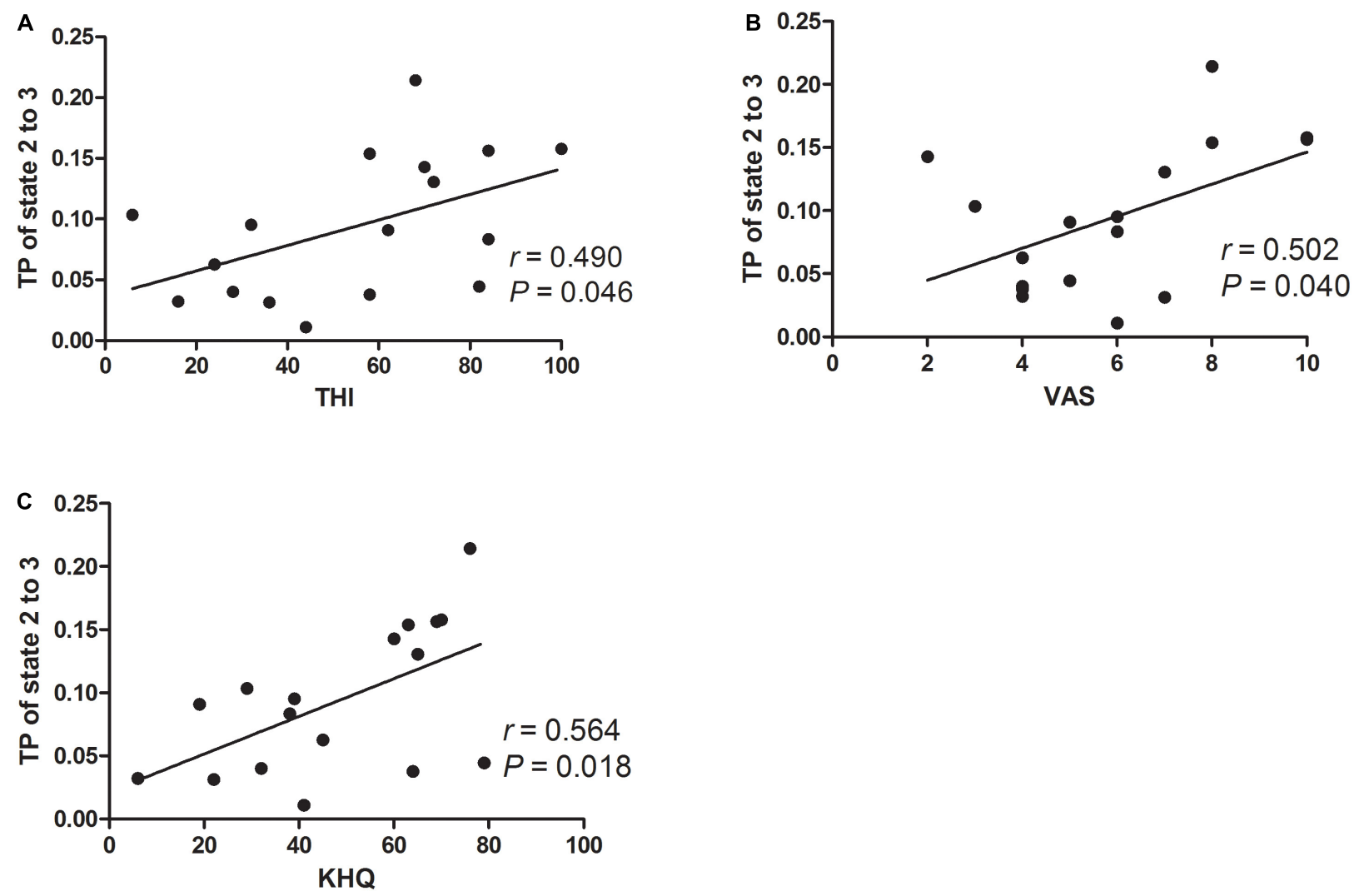

FIGURE 6 | Correlation between TP from state 2 to state 3 and tinnitus severity in chronic tinnitus patients before acupuncture. We found that the TP from state 2 to state 3 in chronic tinnitus patients before acupuncture positively correlated with THI (A), VAS (B), and KHQ (C). KHQ, Khalfa hyperacusis questionnaire; THI, tinnitus handicap inventory; TP, transition probability; VAS, visual analog scales.

loss of the right ear. Moreover, clustering analysis showed higher TP between brain connection states in chronic tinnitus patients, which was positively correlated with tinnitus severity. Consistent with recent studies (Cai et al., 2019; Pang et al., 2019; Kuzucu and Karaca, 2020), the acupuncture treatment effectively improved tinnitus. DFC between the left PCG and right AG in chronic tinnitus patients after acupuncture were observed to significantly decrease, and the amount was positively correlated with the improvement of tinnitus. Clustering analysis showed acupuncture treatment might promote chronic tinnitus patients under lower DFC state, which was also positively correlated with the improvement of tinnitus.

This study found higher temporal variability of DFC between the SMA and SFGmed in chronic tinnitus patients. The previous studies indicated that the SMA and the SFGmed tightly associated with tinnitus (Chen et al., 2016, Cheng et al., 2020). The previous study showed that effective connectivity from the SFG to the SMA was higher in chronic tinnitus patients compared with normal subjects (Chen et al., 2016). Moreover, the meta-analysis showed that tinnitus patients showed structural abnormality in the SMA and the SFG (Cheng et al., 2020). The abnormal activity in the SFG for tinnitus patients might correlate with the emotional problem produced by tinnitus (Golm et al., 2013). Moreover, the previous study suggested that repetitive transcranial magnetic stimulation (rTMS) of the auditory cortex for normal subjects produced the brain activation of the motor cortex (Lee et al., 2013), and rTMS on motor cortex plasticity for a large sample of tinnitus patients decreased short-interval intra-cortical inhibition that might reflect modulation of GABAergic mechanisms directly or indirectly related to rTMS treatment effects (Schecklmann et al., 2014). Therefore, the change of brain functional network between the SMA and SFGmed might be useful to guide the rTMS treatment for tinnitus. Higher time variability of DFC between the left SMA and left SFGmed and its association with hearing loss in chronic tinnitus patients suggest that decreased time variability of DFC might improve the hearing level of chronic tinnitus patients.

The result in this study showed that the temporal variability of DFC between the left PCG and right AG was significantly decreased in chronic tinnitus patients after acupuncture, which was positively correlated with the improvement of tinnitus. Acupuncture might decrease the time variability of DFC between the left PCG and right AG in chronic tinnitus patients that positively correlated with the improvement of tinnitus (TDI scores). This study found TDI and VAS scales scores decreased in chronic tinnitus patients after acupuncture, which is consistent with the previous study (Kim et al., 2017; Kuzucu and Karaca, 2020). The above results indicated that the neural mechanism of 

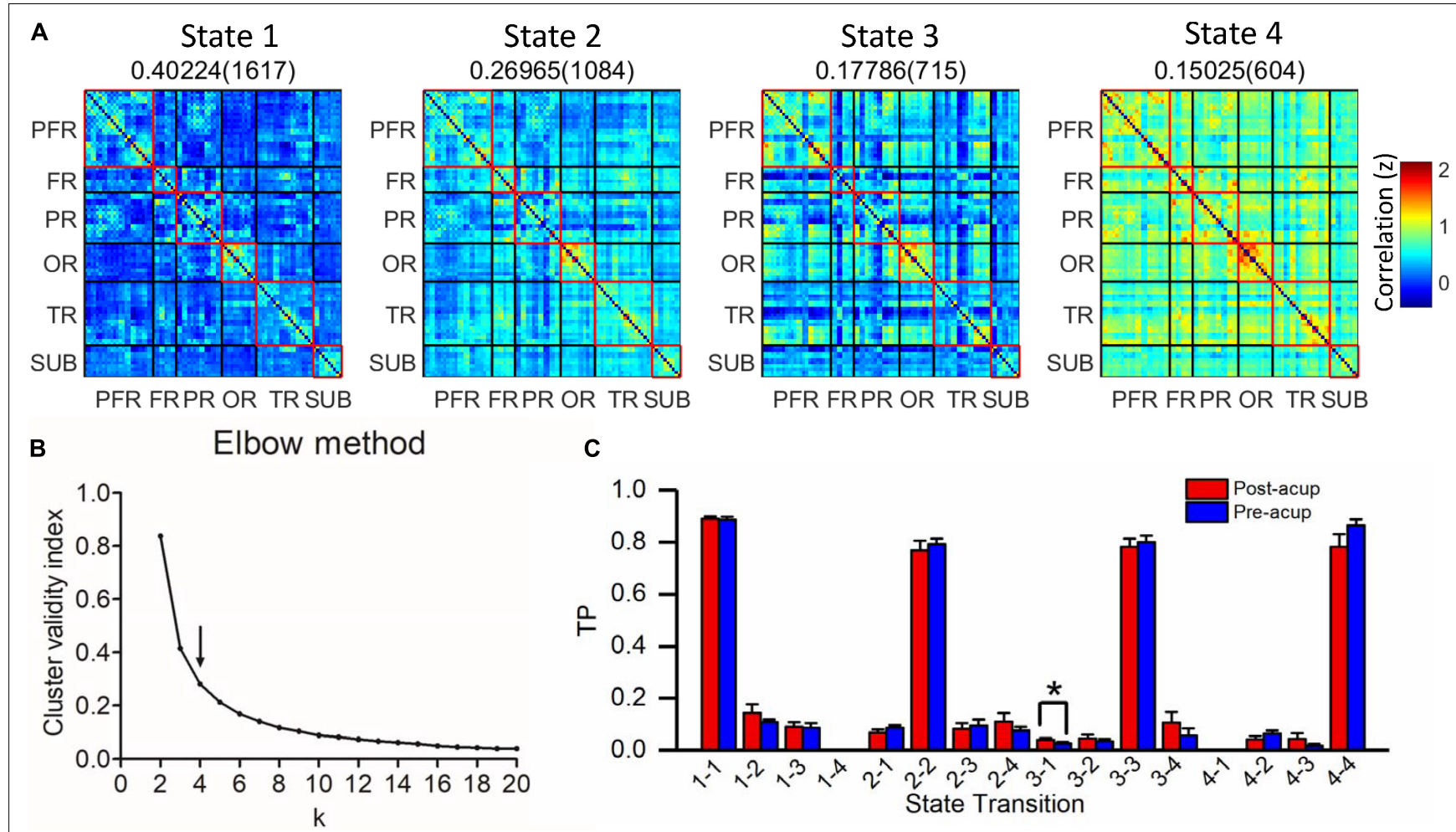

C

FIGURE 7 | Clustering analysis of four states in chronic tinnitus patients before and after acupuncture. (A) Four states (centroids) from clustering analysis. The total number (in brackets) and proportion of occurrences are listed above each state. (B) Elbow criterion. The arrow represents the optimal $k$ value. $k$, the number of clusters; Cluster validity index, calculating as the ratio of within-cluster distance to between-cluster distance. (C) Comparison of TP within chronic tinnitus patients before and after acupuncture. FR, other regions of frontal lobe; PR, parietal regions; OR, occipital regions; PFR, prefrontal regions; SUB, subcortex regions; TP, transition probability; TR, temporal regions. ${ }^{*} P<0.05$.

acupuncture treatment improving tinnitus might be decreasing the time variability of DFC between the left PCG and right AG. The previous studies also indicated that the PCG and the AG tightly associated with chronic tinnitus (Chen et al., 2014, 2015b; Krick et al., 2017). Impaired visual attention function was found in chronic tinnitus patients, and Heidelberg Neuro-Music Therapy treatment helped shift the attention from the auditory phantom percept toward visual cues in chronic tinnitus patients through the AG (Krick et al., 2017). Moreover, the spontaneous brain activity of the right AG, SFG, and medial temporal gyrus in chronic tinnitus patients was higher than that in normal subjects and correlated with tinnitus severity (Chen et al., 2014). Tinnitus patients showed stronger activations to tinnitusrelated sentences in comparison to neutral sentences than healthy controls in various limbic/emotional processing areas, such as the anterior cingulate cortex, middle cingulate cortex, PCG, retrosplenial cortex and insula and also in frontal areas, and the fronto-parietal-cingulate network associated with tinnitusrelated distress (Golm et al., 2013). Meanwhile, researchers indicated that tinnitus duration and distress were associated with brain areas (right inferior frontal gyrus, right ventromedial prefrontal lobe, and right PCG) involved in attentional and emotional processing (Schecklmann et al., 2013). The results in this study suggest that acupuncture might modulate attentional- and emotional-related brain areas to decrease the tinnitus severity.
It's noteworthy that the clustering analysis of DFC suggests that acupuncture treatment may improve tinnitus under hypo-connected pattern. Higher TP from state 2 to state 3 was found in chronic tinnitus patients before acupuncture compared with normal subjects, and increased TP from state 3 (hyper-connected pattern) to state 1 (hypo-connected pattern) was found in chronic tinnitus patients after acupuncture compared with before acupuncture. No significant difference of TP from state 2 to state 3 was found in chronic tinnitus patients after acupuncture compared with before acupuncture. However, acupuncture might improve tinnitus by transitioning state 3 (hyper-connected pattern) to state 1 (hypo-connected pattern). Combining the data of chronic tinnitus patients before and after acupuncture, we found TP from state 3 to state 1 negatively correlated with the tinnitus severity.

This study helps find the objective indicator to assess tinnitus by comparing the brain activity between chronic tinnitus and normal subjects and within chronic tinnitus patients before and after acupuncture. Compared with SFC, DFC may be more suitable as an objective indicator for assessing tinnitus. Compared with normal subjects, the time variability of DFC between the left SMA and left SFGmed in chronic tinnitus patients was higher, which positively correlated with hearing loss of the right ear. Moreover, DFC between the left PCG and right AG in chronic tinnitus patients 
after acupuncture showed as significantly decreased, which was positively correlated with the improvement of tinnitus. No significant difference for SFC after FDR correction was found between chronic tinnitus patients before acupuncture and normal subjects, which is inconsistent with previous studies (Chen et al., 2015a; Hinkley et al., 2015; Lv et al., 2017). This inconsistency might be attributable to a variety of factors: ROIs selection, heterogeneous samples, and different preprocessing methods. Instead of using the AAL atlas, seeds in many studies were defined by prior knowledge (Chen et al., 2015a), some tinnitus-related areas using other atlas (Hinkley et al., 2015; Chen et al., 2017), or independent component analysis (Leaver et al., 2016). Although SFC between the left PCC and left ANG in chronic tinnitus patients after acupuncture was also decreased compared with before acupuncture, no significant correlation was found between SFC of the left PCC and left ANG and tinnitus-related scales scores or hearing level.

There are some limitations in this study. First, the sample size is small. In fact, we spent 2 years (from December 2017 to October 2019) to recruit 33 tinnitus patients and 22 normal subjects. In these patients, we excluded six acute tinnitus patients, and 10 tinnitus patients withdrew consent. Therefore, chronic tinnitus patients in this study were wellcharacterized. Second, no sham treatment or other interventions for normal subjects and chronic tinnitus patients was designed. The result in this study showed that the temporal variability of DFC between the left PCG (belonging to the limbic system) and right $A G$ was significantly decreased in chronic tinnitus patients after acupuncture, which was positively correlated with the improvement of tinnitus. Moreover, the previous study showed that acupuncture modulated the limbic system in healthy subjects (Hui et al., 2000), which is consistent with the findings in our study. On the other hand, there are significant problems with sham interventions and how they are applied in trials of acupuncture, which is caused by paucity of physiological investigations of acupuncture (Birch et al., 2022). Acupuncture is a clinical subject that is safe and effective for the treatment of disease, which has been confirmed by many large authoritative clinical observations (Chang et al., 2019; He et al., 2020). In this study, we compared the FC of chronic tinnitus with normal subjects and showed preliminary results for acupuncture treatment of chronic tinnitus. Future studies should increase the sample size and design sham acupuncture

\section{REFERENCES}

Allen, E. A., Damaraju, E., Plis, S. M., Erhardt, E. B., Eichele, T., and Calhoun, V. D. (2014). Tracking whole-brain connectivity dynamics in the resting state. Cereb. Cortex 24, 663-676. doi: 10.1093/cercor/bhs352

Baguley, D., McFerran, D., and Hall, D. (2013). Tinnitus. Lancet 382, 1600-1607. doi: 10.1016/S0140-6736(13)60142-7

Baguley, D. M., Humphriss, R. L., and Hodgson, C. A. (2000). Convergent validity of the tinnitus handicap inventory and the tinnitus questionnaire. J. Laryngol. Otol. 114, 840-843. doi: 10.1258/0022215001904392

Bauer, C. A., Kurt, W., Sybert, L. T., and Brozoski, T. J. (2013). The cerebellum as a novel tinnitus generator. Hear. Res. 295, 130-139. doi: 10.1016/j.heares.2012. 03.009 group to make the effectiveness of acupuncture treatment for tinnitus more reliable.

\section{CONCLUSION}

As an alternative therapy method, acupuncture might improve tinnitus by decreasing the time variability of DFC in chronic tinnitus patients. Furthermore, this study might provide the neurophysiological evidence for acupuncture treatment of tinnitus clinically.

\section{DATA AVAILABILITY STATEMENT}

The original contributions presented in the study are included in the article, further inquiries can be directed to the corresponding author/s.

\section{ETHICS STATEMENT}

The studies involving human participants were reviewed and approved by the Ethics Committee of the First Affiliated Hospital of Anhui University of Traditional Chinese Medicine. The patients/participants provided their written informed consent to participate in this study.

\section{AUTHOR CONTRIBUTIONS}

BQ, JY, XY, and YRW contributed to the conception of the study. YRW, WZ, XL, and YMW performed the experiment. YRW and YL performed the data analyses. YRW wrote the manuscript. $\mathrm{BZ}, \mathrm{SH}, \mathrm{XW}, \mathrm{XY}, \mathrm{JY}$, and BQ helped perform the analysis with constructive discussions. All authors contributed to the article and approved the submitted version.

\section{FUNDING}

This work was supported by the National Natural Science Foundation of China (Grant Nos. 91859121, 81701665, and 21876041) and the Health Commission of Anhui Province (Grant No. AHWJ2021a019).

Berlot, E., Arts, R., Smit, J., George, E., Gulban, O. F., Moerel, M., et al. (2020). A 7 Tesla fMRI investigation of human tinnitus percept in cortical and subcortical auditory areas. Neuroimage Clin. 25:102166. doi: 10.1016/j.nicl.2020.10 2166

Bhatt, J. M., Lin, H. W., and Bhattacharyya, N. (2016). Prevalence, Severity, Exposures, and Treatment Patterns of Tinnitus in the United States. JAMA Otolaryngol. Head Neck Surg. 142, 959-965. doi: 10.1001/jamaoto.2016.1700

Birch, S., Lee, M. S., Kim, T. H., and Alraek, T. (2022). Historical perspectives on using sham acupuncture in acupuncture clinical trials. Integr. Med. Res. 11:100725. doi: 10.1016/j.imr.2021.100725

Cai, W., Chen, A. W., Ding, L., and Shen, W. D. (2019). Thermal effects of acupuncture by the infrared thermography test in patients with tinnitus. J. Acupunct. Meridian Stud. 12, 131-135. doi: 10.1016/j.jams.2019.05.002 
Chang, S., Kim, D. H., Jang, E. Y., Yoon, S. S., Gwak, Y. S., Yi, Y. J., et al. (2019). Acupuncture attenuates alcohol dependence through activation of endorphinergic input to the nucleus accumbens from the arcuate nucleus. Sci. Adv. 5:eaax1342. doi: 10.1126/sciadv.aax1342

Chen, H. J., Qi, R., Ke, J., Qiu, J., Xu, Q., Zhang, Z., et al. (2020a). Altered dynamic parahippocampus functional connectivity in patients with post-traumatic stress disorder. World J. Biol. Psychiatry 22, 236-245. doi: 10.1080/15622975.2020. 1785006

Chen, Y., Cui, Q., Xie, A., Pang, Y., Sheng, W., Tang, Q., et al. (2020b). Abnormal dynamic functional connectivity density in patients with generalized anxiety disorder. J. Affect. Disord. 261, 49-57. doi: 10.1016/j.jad.2019.09.084

Chen, Y. C., Feng, Y., Xu, J. J., Mao, C. N., Xia, W., Ren, J., et al. (2016). Disrupted brain functional network architecture in chronic tinnitus patients. Front. Aging Neurosci. 8:174. doi: 10.3389/fnagi.2016.00174

Chen, Y. C., Li, X., Liu, L., Wang, J., Lu, C. Q., Yang, M., et al. (2015a). Tinnitus and hyperacusis involve hyperactivity and enhanced connectivity in auditorylimbic-arousal-cerebellar network. Elife 4:e06576. doi: 10.7554/eLife.06576

Chen, Y. C., Xia, W., Chen, H., Feng, Y., Xu, J. J., Gu, J. P., et al. (2017). Tinnitus distress is linked to enhanced resting-state functional connectivity from the limbic system to the auditory cortex. Hum. Brain Mapp. 38, 2384-2397. doi: 10.1002/hbm.23525

Chen, Y. C., Xia, W., Luo, B., Muthaiah, V. P., Xiong, Z., Zhang, J., et al. (2015b). Frequency-specific alternations in the amplitude of low-frequency fluctuations in chronic tinnitus. Front. Neural. Circuits 9:67. doi: 10.3389/fncir. 2015.00067

Chen, Y. C., Zhang, J., Li, X. W., Xia, W., Feng, X., Gao, B., et al. (2014). Aberrant spontaneous brain activity in chronic tinnitus patients revealed by resting-state functional MRI. Neuroimage Clin. 6, 222-228. doi: 10.1016/j.nicl.2014.09.011

Cheng, S., Xu, G., Zhou, J., Qu, Y., Li, Z., He, Z., et al. (2020). A multimodal metaanalysis of structural and functional changes in the brain of tinnitus. Front. Hum. Neurosci. 14:28. doi: 10.3389/fnhum.2020.00028

de Azevedo, R. F., Chiari, B. M., Okada, D. M., and Onishi, E. T. (2007). Impact of acupuncture on otoacoustic emissions in patients with tinnitus. Braz. J. Otorhinolaryngol. 73, 599-607.

Demirtas, M., Tornador, C., Falcon, C., Lopez-Sola, M., Hernandez-Ribas, R., Pujol, J., et al. (2016). Dynamic functional connectivity reveals altered variability in functional connectivity among patients with major depressive disorder. Hum. Brain Mapp. 37, 2918-2930. doi: 10.1002/hbm.23215

Feng, Y., Chen, Y. C., Lv, H., Xia, W., Mao, C. N., Bo, F., et al. (2018). Increased resting-state cerebellar-cerebral functional connectivity underlying chronic tinnitus. Front. Aging Neurosci. 10:59. doi: 10.3389/fnagi.2018.00059

Fox, M. D., Corbetta, M., Snyder, A. Z., Vincent, J. L., and Raichle, M. E. (2006). Spontaneous neuronal activity distinguishes human dorsal and ventral attention systems. Proc. Natl. Acad. Sci. U.S.A. 103, 10046-10051. doi: 10.1073/ pnas.0604187103

Friston, K. J., Williams, S., Howard, R., Frackowiak, R. S., and Turner, R. (1996). Movement-related effects in fMRI time-series. Magn. Reson. Med. 35, 346-355. doi: $10.1002 / \mathrm{mrm} .1910350312$

Golm, D., Schmidt-Samoa, C., Dechent, P., and Kroner-Herwig, B. (2013). Neural correlates of tinnitus related distress: an fMRI-study. Hear. Res. 295, 87-99. doi: 10.1016/j.heares.2012.03.003

Greicius, M. D., Supekar, K., Menon, V., and Dougherty, R. F. (2009). Restingstate functional connectivity reflects structural connectivity in the default mode network. Cereb. Cortex 19, 72-78. doi: 10.1093/cercor/bhn059

Guo, S., Zhao, W., Tao, H., Liu, Z., and Palaniyappan, L. (2018). The instability of functional connectivity in patients with schizophrenia and their siblings: a dynamic connectivity study. Schizophr. Res. 195, 183-189. doi: 10.1016/j.schres. 2017.09.035

Han, Y., Wang, J., Zhao, Z., Min, B., Lu, J., Li, K., et al. (2011). Frequencydependent changes in the amplitude of low-frequency fluctuations in amnestic mild cognitive impairment: a resting-state fMRI study. Neuroimage 55, 287295. doi: 10.1016/j.neuroimage.2010.11.059

He, C., Chen, Y., Jian, T., Chen, H., Guo, X., Wang, J., et al. (2018). Dynamic functional connectivity analysis reveals decreased variability of the defaultmode network in developing autistic brain. Autism Res. 11, 1479-1493. doi: 10.1002/aur.2020

He, Y., Guo, X., May, B. H., Zhang, A. L., Liu, Y., Lu, C., et al. (2020). Clinical evidence for association of acupuncture and acupressure with improved cancer pain: a systematic review and meta-analysis. JAMA Oncol. 6, 271-278. doi: 10.1001/jamaoncol.2019.5233

Hinkley, L. B., Mizuiri, D., Hong, O., Nagarajan, S. S., and Cheung, S. W. (2015). Increased striatal functional connectivity with auditory cortex in tinnitus. Front. Hum. Neurosci. 9:568. doi: 10.3389/fnhum.2015.00568

Huang, K., Liang, S., Chen, L., and Grellet, A. (2021). Acupuncture for tinnitus: a systematic review and meta-analysis of randomized controlled trials. Acupunct. Med. 39, 264-271. doi: 10.1177/0964528420938380

Hui, K. K., Liu, J., Makris, N., Gollub, R. L., Chen, A. J., Moore, C. I., et al. (2000). Acupuncture modulates the limbic system and subcortical gray structures of the human brain: evidence from fMRI studies in normal subjects. Hum. Brain Mapp. 9, 13-25.

Hui, K. K., Liu, J., Marina, O., Napadow, V., Haselgrove, C., Kwong, K. K., et al. (2005). The integrated response of the human cerebro-cerebellar and limbic systems to acupuncture stimulation at ST 36 as evidenced by fMRI. Neuroimage 27, 479-496. doi: 10.1016/j.neuroimage.2005.04.037

Jeon, S. W., Kim, K. S., and Nam, H. J. (2012). Long-term effect of acupuncture for treatment of tinnitus: a randomized, patient- and assessor-blind, shamacupuncture-controlled, pilot trial. J. Altern. Complement. Med. 18, 693-699. doi: $10.1089 / \mathrm{acm} .2011 .0378$

Khalfa, S., Dubal, S., Veuillet, E., Perez-Diaz, F., Jouvent, R., and Collet, L. (2002). Psychometric normalization of a hyperacusis questionnaire. ORL J. Otorhinolaryngol. Relat. Spec. 64, 436-442.

Kim, B. H., Kim, K., and Nam, H. J. (2017). A comparative study on the effects of systemic manual acupuncture, periauricular electroacupuncture, and digital electroacupuncture to treat tinnitus: a randomized, paralleled, open-labeled exploratory trial. BMC Complement. Altern. Med. 17:85. doi: 10.1186/s12906017-1589-3

Kim, J. I., Choi, J. Y., Lee, D. H., Choi, T. Y., Lee, M. S., and Ernst, E. (2012). Acupuncture for the treatment of tinnitus: a systematic review of randomized clinical trials. BMC Complement. Altern. Med. 12:97. doi: 10.1186/1472-688212-97

Krick, C. M., Argstatter, H., Grapp, M., Plinkert, P. K., and Reith, W. (2017). Heidelberg neuro-music therapy restores attention-related activity in the angular gyrus in chronic tinnitus patients. Front. Neurosci. 11:418. doi: 10.3389/ fnins.2017.00418

Kuzucu, I., and Karaca, O. (2020). Acupuncture treatment in patients with chronic subjective tinnitus: a prospective, randomized study. Med. Acupunct. 32, 24-28. doi: $10.1089 /$ acu.2019.1367

Lan, L., Li, J., Chen, Y., Chen, W., Li, W., Zhao, F., et al. (2020). Alterations of brain activity and functional connectivity in transition from acute to chronic tinnitus. Hum. Brain Mapp. 42, 485-494. doi: 10.1002/hbm.25238

Laureano, M. R., Onishi, E. T., Bressan, R. A., Neto, P. B., Castiglioni, M. L. I, Batista, R., et al. (2016). The effectiveness of acupuncture as a treatment for tinnitus: a randomized controlled trial using $(99 \mathrm{~m})$ Tc-ECD SPECT. Eur. Radiol. 26, 3234-3242. doi: 10.1007/s00330-015-4164-7

Leaver, A. M., Turesky, T. K., Seydell-Greenwald, A., Morgan, S., Kim, H. J., and Rauschecker, J. P. (2016). Intrinsic network activity in tinnitus investigated using functional MRI. Hum. Brain Mapp. 37, 2717-2735. doi: 10.1002/hbm. 23204

Lee, M., Kim, S. E., Kim, W. S., Han, J., Kim, H. J., Kim, B. S., et al. (2013). Cortico-cortical modulation induced by $1-\mathrm{Hz}$ repetitive transcranial magnetic stimulation of the temporal cortex. J. Clin. Neurol. 9, 75-82. doi: 10.3988/jcn. 2013.9.2.75

Leonardi, N., and Van De Ville, D. (2015). On spurious and real fluctuations of dynamic functional connectivity during rest. Neuroimage 104, 430-436. doi: 10.1016/j.neuroimage.2014.09.007

Li, Y., Zhu, Y., Nguchu, B. A., Wang, Y., Wang, H., Qiu, B., et al. (2019). Dynamic functional connectivity reveals abnormal variability and hyperconnected pattern in autism spectrum disorder. Autism Res. 13, 230-243. doi: 10.1002/aur.2212

Lin, T. Y., Yang, S. W., Lee, Y. S., Wu, P. W., Young, C. K., Li, T. H., et al. (2019). Analysis of factors influencing the efficiency of acupuncture in tinnitus patients. Evid. Based Complement. Alternat. Med. 2019:1318639. doi: 10.1155/ 2019/1318639

Lindberg, P., Scott, B., Lyttkens, L., and Melin, L. (1992). Multiple assessment of tinnitus through the use of portable matching equipment and self-recordings on visual analog scales. Tinnitus $91,53-56$. 
Liu, F., Wang, Y., Li, M., Wang, W., Li, R., Zhang, Z., et al. (2017). Dynamic functional network connectivity in idiopathic generalized epilepsy with generalized tonic-clonic seizure. Hum. Brain Mapp. 38, 957-973. doi: 10.1002/ hbm. 23430

Liu, J., Liao, X., Xia, M., and He, Y. (2018). Chronnectome fingerprinting: identifying individuals and predicting higher cognitive functions using dynamic brain connectivity patterns. Hum. Brain Mapp. 39, 902-915. doi: 10.1002/hbm. 23890

Liu, X. P., and Chen, L. (2012). Auditory brainstem response as a possible objective indicator for salicylate-induced tinnitus in rats. Brain Res. 1485, 88-94. doi: 10.1016/j.brainres.2012.04.048

Lloyd, S. (1982). Least squares quantization in PCM. IEEE Trans. Inform. Theory 28, 129-137.

Lockwood, A. H., Salvi, R. J., Coad, M. L., Towsley, M. L., Wack, D. S., and Murphy, B. W. (1998). The functional neuroanatomy of tinnitus: evidence for limbic system links and neural plasticity. Neurology 50, 114-120.

Lv, H., Chen, Q., Wei, X., Liu, C., Zhao, P., Wang, Z., et al. (2021). Sound therapy can modulate the functional connectivity of the auditory network. Prog. Neuropsychopharmacol. Biol. Psychiatry 110:110323. doi: 10.1016/j.pnpbp.2021. 110323

Lv, H., Zhao, P., Liu, Z., Li, R., Zhang, L., Wang, P., et al. (2017). Abnormal regional activity and functional connectivity in resting-state brain networks associated with etiology confirmed unilateral pulsatile tinnitus in the early stage of disease. Hear. Res. 346, 55-61. doi: 10.1016/j.heares.2017.02.004

Marschall, T. M., Curcic-Blake, B., Brederoo, S. G., Renken, R. J., Linszen, M. M. J., Koops, S., et al. (2021). Spontaneous brain activity underlying auditory hallucinations in the hearing-impaired. Cortex 136, 1-13. doi: 10.1016/j.cortex. 2020.12.005

Napadow, V., Dhond, R., Park, K., Kim, J., Makris, N., Kwong, K. K., et al. (2009). Time-variant fMRI activity in the brainstem and higher structures in response to acupuncture. Neuroimage 47, 289-301. doi: 10.1016/j.neuroimage.2009.0 3.060

Newman, C. W., Jacobson, G. P., and Spitzer, J. B. (1996). Development of the tinnitus handicap inventory. Arch. Otolaryngol. Head Neck Surg. 122, 143-148.

Pang, P., Shi, Y., Xu, H., Deng, L., Wu, S., and Chen, X. (2019). Acupuncture methods put to the test for a tinnitus study: a Bayesian analysis. Complement. Ther. Med. 42, 205-213. doi: 10.1016/j.ctim.2018.11.017

Pascual-Marqui, R. D., Michel, C. M., and Lehmann, D. (1995). Segmentation of brain electrical activity into microstates: model estimation and validation. IEEE Trans. Biomed. Eng. 42, 658-665.

Peng, L., Mu, K., Liu, A., Zhou, L., Gao, Y. I, Shenoy, T., et al. (2018). Transauricular vagus nerve stimulation at auricular acupoints Kindey (CO10), Yidan (CO11), Liver (CO12) and Shenmen (TF4) can induce auditory and limbic cortices activation measured by fMRI. Hear. Res. 359, 1-12. doi: 10.1016/j.heares.2017. 12.003

Podoshin, L., Ben-David, Y., Fradis, M., Gerstel, R., and Felner, H. (1991). Idiopathic subjective tinnitus treated by biofeedback, acupuncture and drug therapy. Ear. Nose Throat. J. 70, 284-289.

Qin, J., Chen, S. G., Hu, D. W., Zeng, L. L., Fan, Y. M., Chen, X. P., et al. (2015). Predicting individual brain maturity using dynamic functional connectivity. Front. Hum. Neurosci. 9:418. doi: 10.3389/fnhum.2015.00418

Schecklmann, M., Landgrebe, M., Kleinjung, T., Frank, E., Sand, P. G., Rupprecht, R., et al. (2014). Changes in motor cortex excitability associated with temporal repetitive transcranial magnetic stimulation in tinnitus: hints for cross-modal plasticity? BMC Neurosci. 15:71. doi: 10.1186/1471-2202-15-71

Schecklmann, M., Landgrebe, M., Poeppl, T. B., Kreuzer, P., Manner, P., Marienhagen, J., et al. (2013). Neural correlates of tinnitus duration and distress: a positron emission tomography study. Hum. Brain Mapp. 34, 233-240. doi: $10.1002 / \mathrm{hbm} .21426$
Seeley, W. W., Menon, V., Schatzberg, A. F., Keller, J., Glover, G. H., Kenna, H., et al. (2007). Dissociable intrinsic connectivity networks for salience processing and executive control. J. Neurosci. 27, 2349-2356. doi: 10.1523/JNEUROSCI. 5587-06.2007

Tunkel, D. E., Bauer, C. A., Sun, G. H., Rosenfeld, R. M., Chandrasekhar, S. S., Cunningham, E. R. Jr., et al. (2014). Clinical practice guideline: tinnitus. Otolaryngol. Head Neck Surg. 151 (2 Suppl), S1-S40. doi: 10.1177/ 0194599814545325

Tzourio-Mazoyer, N., Landeau, B., Papathanassiou, D., Crivello, F., Etard, O., Delcroix, N., et al. (2002). Automated anatomical labeling of activations in SPM using a macroscopic anatomical parcellation of the MNI MRI single-subject brain. Neuroimage 15, 273-289. doi: 10.1006/nimg.2001.0978

Wang, C., Ong, J. L., Patanaik, A., Zhou, J., and Chee, M. W. (2016). Spontaneous eyelid closures link vigilance fluctuation with fMRI dynamic connectivity states. Proc. Natl. Acad. Sci. U.S.A. 113, 9653-9658. doi: 10.1073/pnas.1523980113

Wang, J., Wang, X., Xia, M., Liao, X., Evans, A., and He, Y. (2015). GRETNA: a graph theoretical network analysis toolbox for imaging connectomics. Front. Hum. Neurosci. 9:386. doi: 10.3389/fnhum.2015.00386

Wang, R., Sun, C., Lin, J., Chen, N., Hu, B., Liu, X., et al. (2020). Altered dynamic functional connectivity in patients with mitochondrial encephalomyopathy with lactic acidosis and stroke-like episodes (MELAS) at acute and chronic stages: shared and specific brain connectivity abnormalities. J. Magn. Reson. Imaging 53, 427-436. doi: 10.1002/jmri.27353

Wang, S., Chen, Z., Dai, J., Yu, F., and Kang, H. (2021). Evaluation of the therapeutic effect of acoustic therapy combined with acupuncture on idiopathic tinnitus: a randomized controlled trial protocol. Medicine (Baltimore) 100:e23883. doi: 10.1097/MD.0000000000023883

Xie, H., Li, X., Lai, J., Zhou, Y., Wang, C., and Liang, J. (2014). Effectiveness of De Qi during acupuncture for the treatment of tinnitus: study protocol for a randomized controlled trial. Trials 15:397. doi: 10.1186/17456215-15-397

Yan, C. G., and Zang, Y. F. (2010). DPARSF: a MATLAB toolbox for pipeline data analysis of resting-state fMRI. Front. Syst. Neurosci. 4:13. doi: 10.3389/fnsys. 2010.00013

Yang, H., Cai, Y., Guo, H., Xiong, H., Sun, Y., Huang, X., et al. (2018). Prevalence and factors associated with tinnitus: data from adult residents in Guangdong province, South of China. Int. J. Audiol. 57, 892-899. doi: 10.1080/14992027. 2018.1506169

Zalesky, A., Fornito, A., Cocchi, L., Gollo, L. L., and Breakspear, M. (2014). Time-resolved resting-state brain networks. Proc. Natl. Acad. Sci. U.S.A. 111, 10341-10346. doi: 10.1073/pnas.1400181111

Conflict of Interest: The authors declare that the research was conducted in the absence of any commercial or financial relationships that could be construed as a potential conflict of interest.

Publisher's Note: All claims expressed in this article are solely those of the authors and do not necessarily represent those of their affiliated organizations, or those of the publisher, the editors and the reviewers. Any product that may be evaluated in this article, or claim that may be made by its manufacturer, is not guaranteed or endorsed by the publisher.

Copyright (C) 2022 Wei, Zhang, Li, Liu, Zha, Hu, Wang, Wang, Yu, Yang and Qiu. This is an open-access article distributed under the terms of the Creative Commons Attribution License (CC BY). The use, distribution or reproduction in other forums is permitted, provided the original author(s) and the copyright owner(s) are credited and that the original publication in this journal is cited, in accordance with accepted academic practice. No use, distribution or reproduction is permitted which does not comply with these terms. 